\title{
Separation control by tangential blowing inside the bubble
}

\author{
P. R. Viswanath, G. Ramesh, K. T. Madhavan
}

\begin{abstract}
Experiments have been carried out investigating the effectiveness of steady tangential blowing (inside the separation bubble) to control an axisymmetric separated flow at low speeds. Turbulent boundary separation was induced on a contoured afterbody and the separated shear layer reattached on a narrow cylindrical sting. Measurements made consisted of model surface pressures, mean velocity, turbulent shear stress and kinetic energy profiles using a 2-component LDV system. The results explicitly demonstrate that blowing downstream of the separation location, but within the bubble, can be an effective means of separation control, considering both wall and wake flow reversals.
\end{abstract}

\section{List of symbols}

$C_{p} \quad$ Static pressure coefficient $\left(=p-p_{\infty} / q_{\infty}\right)$

$C_{\mu} \quad$ Blowing momentum coefficient (2D)

$C_{\mu \mathrm{A}} \quad$ Blowing momentum coefficient (axisymmetric)

D Model forebody diameter

$h \quad$ Blowing slot height

$k \quad\left\langle u^{\prime 2}+v^{\prime 2}\right\rangle / 2$, Turbulent kinetic energy

$m_{\mathrm{bl}} \quad$ Boundary layer mass flow rate

$m_{j} \quad$ Jet mass flow rate

$p \quad$ Local surface pressure

$p_{\infty} \quad$ Freestream static pressure

$q_{\infty} \quad$ Freestream dynamic pressure

$u \quad$ Local velocity in the boundary layer

$U_{\infty} \quad$ Freestream velocity

$U_{j} \quad$ Jet velocity

$\left\langle u^{\prime 2}\right\rangle$ Mean square velocity fluctuation in $x$ direction

$\left\langle v^{\prime 2}\right\rangle \quad$ Mean square velocity fluctuation in $y$ direction

$\tau \quad\left\langle-u^{\prime} v^{\prime}\right\rangle$, Reynolds shear stress

$\delta^{*} \quad$ Boundary layer displacement thickness

$\theta \quad$ Boundary layer momentum thickness

$x \quad$ Coordinate parallel to model axis

$y \quad$ Coordinate normal to model axis

Received: 16 October 1998/Accepted: 27 September 1999

P. R. Viswanath, G. Ramesh, K. T. Madhavan

Experimental Aerodynamics Division

National Aerospace Laboratories, Bangalore 560 017, India

This research was funded by the Aeronautical Research \& Development Board, Govt. of India. One of us (P.R. Viswanath) sincerely thank Prof. Roddam Narasimha and Anatol Roshko for many interesting discussions on this work.

\section{Introduction}

The problem of turbulent boundary layer separation the associated aerodynamic effects have been the subien of numerous investigations in the literature (e.g. Chang 1970; Simpson, 1989). The boundary layer separation is result of strong adverse pressure gradients in the directio of the flow and leads to increased energy losses. The general problem of turbulent boundary layer separation sufficiently complex involving, for example, three-dimen sionality, large-scale unsteadiness that most earlier studis in the literature have focussed attention on nominally tur dimensional separated flows (e.g. Stratford, 1959; Sand. born and Liu, 1968; Simpson et al., 1981; Viswanath an: Brown, 1983; Simpson, 1985; Thompson and Whitelaw, 1985; Viswanath, 1988). While significant developments have taken place during the last decade in the calculatin methods for separated flows, difficulty in modelling tur: bulent stresses in such flows still remains (Marvin, 1993 Carefully planned building-block experiments involving measurements of mean as well as turbulence quantities have been of great value in improving aspects of turbu. lence modelling for separated flows (Marvin, 1982, 1987 Simpson, 1987; Williams, 1989).

Separation control by passive or active means is wide employed for improving aerodynamic performance. The monograph by Chang (1976) contains an excellent accous of several passive and active methods of separation contr in different speed regimes. Recent reviews on the subje include that of Gad-El-Hak and Bushnell (1991) and Wygnanski (1997). Tangential blowing, which involves injection of fluid parallel to the wall through a narrow st: is generally known to be an effective means of separatio control (Peake, 1966; Viswanath, 1988; Wong, 1977); th: injected mass energizes the boundary layer near the wa providing sufficient kinetic energy to negotiate adverse pressure gradients. Since blowing involves injection of additional mass and momentum into the boundary lay the parameters affecting its performance include the velocity, density and the slot height (in two-dimension flows). The most widely used and relevant parameter (Lachmann, 1961; Chang, 1976) is the blowing momenti coefficient, $C_{\mu}$, defined by

$C_{\mu}=m_{\mathrm{j}} U_{\mathrm{j}} / \rho_{0} U_{0}^{2} \theta_{0}$

where $m_{\mathrm{j}}$ is the jet mass flow rate per unit span, $U_{\mathrm{j}}$ is th velocity, $\rho_{0}$ and $U_{0}$ are the (local) freestream density, locity and $\theta_{0}$ is the boundary layer momentum thicknt 
just ahead of separation. Other definitions of $C_{\mu}$ have been mployed as well in the literature (e.g. Chang, 1976) which ilize different velocity and length scales; for example, cess velocity $\left(U_{\mathrm{j}}-U_{0}\right)$ is used instead of $U_{\mathrm{j}}$ for the omentum injected and a length scale of the body (e.g. aiffoil chord in a 2D flow) is often used for normalization. Here we prefer a viscous scale like $\theta_{0}$ since it would reflect local boundary layer property; as a result, $C_{\mu}$ values so defined, will be generally an order of magnitude larger. Among the several factors that may determine the effectiveness of injection, earlier studies (Peake, 1966; Vismath, 1988) have shown that the injection slot location is critical parameter. It is convenient to distinguish befween two types of injection, depending upon the slot location:

U-type: injection is upstream of separation point (i.e., the conventional location adopted for boundary layer control).

(II) D-type: injection is downstream of separation point, but within the recirculation zone.

Viswanath et al., (1983) discussed briefly some of the difficulties associated with U-type injection in the supersonic case and provided the first assessment of D-type injection effectiveness in a ramp-induced turbulent separated flow at a Mach number of 2.50; based on measurements of surface pressure distributions and limited pitot pressure survey in the interaction region, they showed that D-type injection was more effective than U-type. Beyond these early observations, there has been no attempt in the iterature assessing either the general effectiveness of Dtype injection to other separated flows or its exploitation in design applications.

It is to be recognized that studies involving tangential blowing or injection have been reported in other flow situations; for example, to provide lift enhancement on airfoils and wings using trailing-edge blowing which may involve D-type injection (Lachmann, 1961). In contrast, the emphasis and focus in the present work is on the uppression of boundary layer separation to reduce seerity of viscous effects.

In this paper, we present new results from an experiental programme specifically designed to assess in detail effectiveness of D-type injection in a low speed separated flow. Turbulent boundary layer separation occurred an axisymmetric contoured afterbody due to sustained adverse pressure gradients. Based on surface pressure and detailed flow-field measurements employing a 2-compo- nent LDV system, it is explicitly demonstrated that D-type tangential injection can be an effective means of separation control, considering both wall and wake flow reversals.

\section{2}

\section{Experiments}

\section{1}

\section{Test facility and model configuration}

The experiments were performed in the $0.91 \mathrm{~m}$ dia. low speed wind tunnel at a freestream velocity $\left(U_{\infty}\right)$ of $20 \mathrm{~m} / \mathrm{s}$. The axisymmetric model configuration employed had a diameter $(D)$ of $122 \mathrm{~mm}$ and a total length of $1420 \mathrm{~mm}$ made up in 3 sections: a tangent ogive nose $300 \mathrm{~mm}$ long, a central cylindrical section $1047 \mathrm{~mm}$ long and a circular arc afterbody (of radius of curvature $=128 \mathrm{~mm}$ ) $98 \mathrm{~mm}$ long (Fig. 1); the afterbody also carried a sting of $30 \mathrm{~mm}$ dia. and $330 \mathrm{~mm}$ long on which the separated flow reattached. Two afterbody models were fabricated for making measurements, both with and without flow control.

Figure 1 shows the afterbody model with facility for tangential blowing with an annular axisymmetric slot height (h) $2.5 \mathrm{~mm}$ (Fig. 1): sufficient care was taken in the slot design to ensure tangential injection and the above value of $h$ was chosen primarily from supersonic experience (Viswanath et al., 1983). The slot location $(x=-15 \mathrm{~mm}$ ) was chosen so that it is downstream of the separation point (in the absence of injection) but within the reversed flow zone based on preliminary experiments. The model was supported with a thin rectangular strut at a distance of $300 \mathrm{~mm}$ from the nose which also provided the passage for the jet flow into the model.

\section{2}

\section{Instrumentation and measurements}

Model static pressure distributions on the cylinder and afterbody were measured using Furness Control micromanometers primarily on the lee-ward (top) generator (limited measurements at a few other generators on the afterbody were carried out as well); these measurements were made at three values of blowing or injection velocity $\left(U_{\mathrm{j}}\right)$ of 15,25 and $31 \mathrm{~m} / \mathrm{s}$; the jet mass flow was calculated using a rotometer installed in the jet flow circuit.

The flow measurements of the mean and turbulent quantities were made using a three-beam, two-component DANTEC laser Doppler velocimeter. The LDV was used in the forward scatter mode to achieve higher signal-to-noise ratio. The focal length of the front lens was $600 \mathrm{~mm}$ and

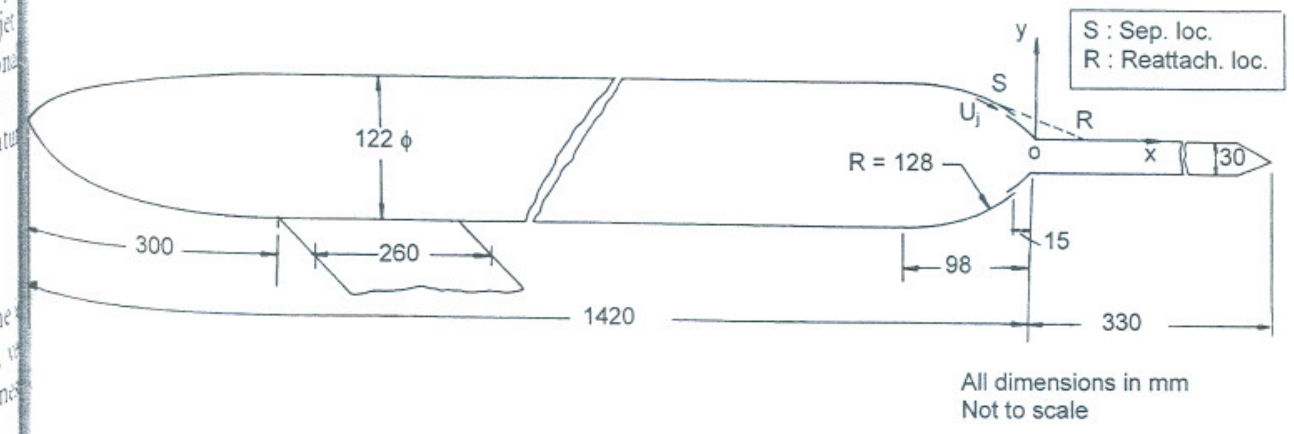

Fig. 1. Schematic of axisymmetric model 
the beam intersection angle was $4.66^{\circ}$. The ellipsoidal probe volume had a length of about $2 \mathrm{~mm}$ and a diameter of $0.2 \mathrm{~mm}$. Fringe spacing in the blue and green components were 6.8 and $6.0 \mu \mathrm{m}$ respectively. A Bragg cell with an optical frequency shift to $40 \mathrm{MHz}$ was used for measuring the reversed flow; in addition, an electronic frequency shift was also used for accurate measurement of low velocities. Data from the counter-type signal processor was transferred to the PC/AT system using DMA-based high-speed digital I/O cards. All the software modules, including those for acquiring Doppler data from DIO of counter processor, LDV parameter selection, quick check of on-line data and processing of data were developed at NAL (Ramesh et al., 1994).

The mean velocity, turbulent shear stress and twocomponent turbulent kinetic energy measurements were made with optics in the $\pm 45^{\circ}$ configuration; limited mean velocity data and intermittency measurements, (characterising the fraction of time the flow is reversed) in the vicinity of separation location were carried out with optics in the $0 / 90^{\circ}$ configuration.

Seeding was accomplished successfully using a smoke generator with liquid paraffin developed in our laboratory. It was found that paraffin smoke provided sufficiently good particle concentration even within the recirculating zones of the flow. No direct estimate of the particle sizes was made; however, we expect that the particle size is in the range of 2 to 5 microns, as reported by Wiedemann (1994). The data rates were typically in the range 400 to $600 \mathrm{samples} / \mathrm{s}$. Data validation rates were in the range of 500 to 800 for a batch of 1000 samples, which is generally indicative of good SNR conditions.

In the present experiments involving measurements in a separated flow, preliminary studies were made to arrive at an optimum ensemble size for determining the statistics in the attached and reversed flow zones. Although 5000 samples were found adequate for obtaining good repeatability of mean velocities in the attached (or forward) flow regions, the quality of the turbulent shear stress and kinetic energy data showed visible improvements with 10,000 samples. So, a minimum ensemble size of 10,000 samples was adopted for obtaining the mean as well as the turbulent quantities in zones with $u>0$. A similar exercise for the reversed flow region $(u<0)$ suggested that an ensemble size of 20,000 samples should be adequate which was finally adopted.

\section{3}

\section{Estimates of uncertainty in the measured data}

For the LDV data, sources of error include optical, statistical and positional. The uncertainty in the measurement of beam half-angle is estimated to be $\pm 0.01^{\circ}$ which translates to an uncertainty in velocity of $\pm 0.05 \mathrm{~m} / \mathrm{s}$; however, the resolution of the burst counter results in a relatively higher uncertainly of $\pm 0.1 \mathrm{~m} / \mathrm{s}$. For the sample size used for each data point (discussed above) we expect the statistical errors to be small $(<1 \%)$. No correction for the measured data for possible velocity bias was made since such corrections did not seem warranted based on comparisons of measurements in certain standard flows as well as redundancy measurements (Ramesh et al., 1995); such an observation has also been made by Admas a Eaton (1988) in low speed separated flow. Furthermo reliable corrections schemes are not established for ve locity bias. Likely errors in the measured mean veloci turbulent shear stress and 2-component kinetic energ data are given below; these estimates are based on re peatability tests and comparison with redundancy mea, surements, wherever possible.

\begin{tabular}{ll}
\hline Quantity & Error \\
\hline Pressure coefficient, $C_{p}$ & $\leq \pm 0.3 \%$ \\
Mean velocity, $u$ & $\leq \pm 2 \%$ or $0.1 \mathrm{~m} / \mathrm{s}$ \\
& $\quad$ whichever is higher \\
Turbulent shear stress $(\tau)$ & $\leq \pm 6 \%$ \\
Turbulent kinetic energy $(k)$ & $\leq \pm 8 \%$ \\
$x$ & $\leq \pm 0.5 \mathrm{~mm}$ \\
$y$ & $\leq \pm 0.3 \mathrm{~mm}$ \\
\hline
\end{tabular}

3

\section{Results and discussion}

\section{1}

\section{Without tangential blowing}

\subsection{1}

\section{Boundary layer properties on the cylinder}

The measured mean velocity profile on the cylinder at $x=-258 \mathrm{~mm}$ showed features of a well developed tur. bulent boundary layer with the following properties (Ramesh et al., 1995):

Boundary layer thickness $(\delta)=25 \mathrm{~mm}$

Displacement thickness $\left(\delta^{*}\right)=3 \mathrm{~mm}$

Momentum thickness $(\theta)=2.3 \mathrm{~mm}$

Wall skin friction coefficient $\left(C_{\mathrm{f}}\right)=0.0036$

\subsection{2}

\section{Surface pressure distributions}

The measured surface $C_{p}$ distributions on the afterbod: and sting along the top generator are shown in Fig. 2( results with blowing will be discussed in Sect. 3.2); the measured $C_{p}$ on two side generators $\left(90^{\circ}\right.$ apart) showe good agreement with those measured on the top genere suggesting good axisymmetry (Ramesh et al., 1995). Th static pressure over a large part of the cylinder is esset tially constant (Ramesh et al., 1995), decreases gradual as the flow expands around the cylinder - afterbody junction due to the change in surface curvature and in followed by a strong adverse pressure gradient leading boundary layer separation as seen by the pressure plate this is followed by a reattachment pressure rise and a pressure decay to freestream value on the sting.

The streamwise development of velocity profiles (Fig. 3), covering all the way upstream of separation 2 downstream of reattachment, show features typical of adverse pressure gradient boundary layer flow eventuz leading to separation; the separation $\left(x_{\mathrm{s}}\right)$ and reattachnt locations $\left(x_{\mathrm{R}}\right)$ are identified at $x=-28 \mathrm{~mm}( \pm 1 \mathrm{~mm})$ 


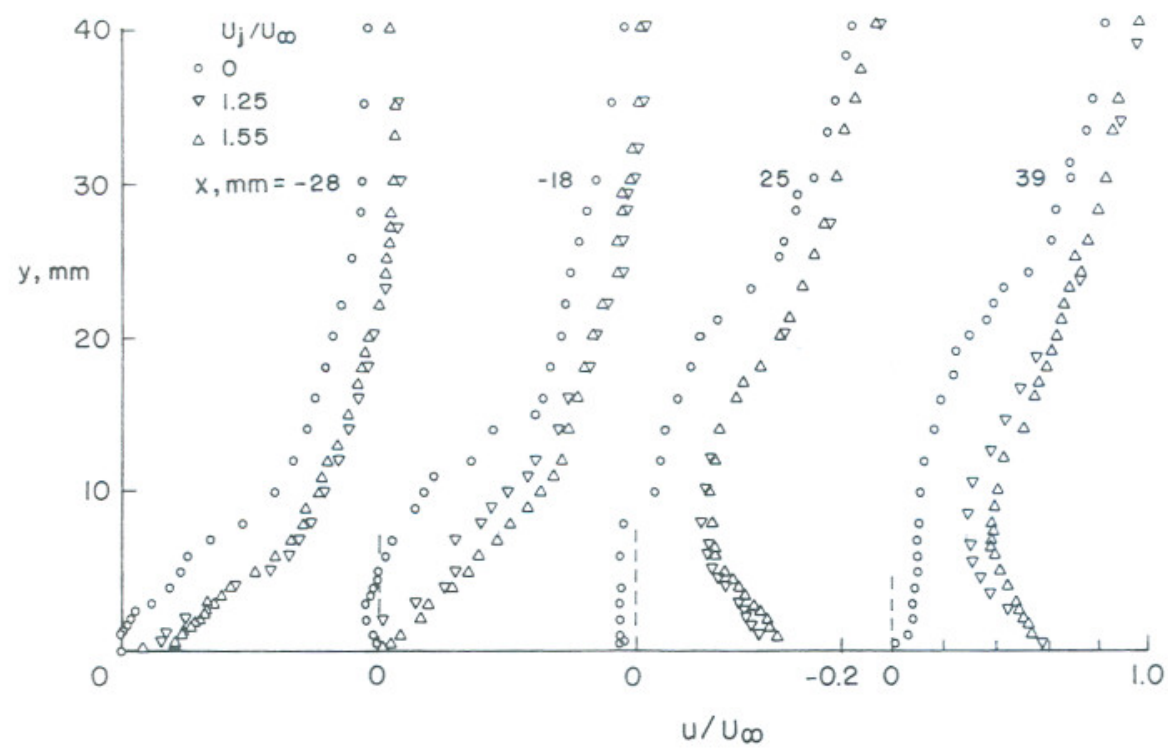

Fig. 5. Effect of blowing on mean velocity profiles of the reversed flow in the separated zone $(x=-18 \mathrm{~mm}$, $25 \mathrm{~mm}$ : Fig. 5) at both values of $U_{\mathrm{j}}\left(=1.25\right.$ and $\left.1.55 U_{\infty}\right)$ is consistent with the observed features of the surface pressure distributions discussed above. At all four stations, increased mean velocities all across the layer suggest efficient mixing of the injected jet with the surrounding flow. The jet mass flow at $U_{j}=25$ and $31 \mathrm{~m} / \mathrm{s}$ correspond to 13 and $16 \%$ (respectively) of the mass flow in the boundary layer at separation $\left(x_{\mathrm{s}}=-28 \mathrm{~mm}\right)$.

\subsection{3}

\section{Boundary layer integral parameters}

The streamwise variations of boundary layer displacement and momentum thickness distributions, corresponding to blowing at $U_{j}=1.55 U_{\infty}$ (and without it) are presented in Fig. 6; as may be expected, due to the elimination of the reversed flow as well as increased mean velocities, both $\delta^{*}$ and $\theta$ values are significantly lower with blowing.

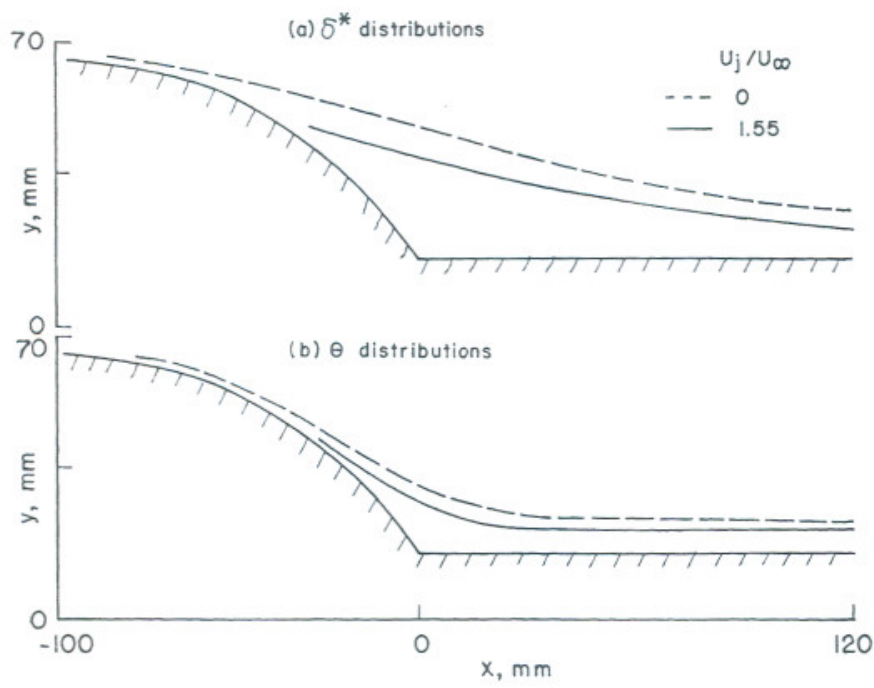

Fig. 6. Boundary layer displacement and momentum thickness distributions

\subsection{4}

Turbulent shear stress and kinetic energy profiles

The variations of the normalised turbulent shear strest and 2-component turbulent kinetic energy profiles at ti highest jet velocity ratio of 1.55 are presented in Figs. and 8 respectively. The complex qualitative nature of t shear stress profiles can be explained from the corresponding mean velocity profiles shown in Fig. 5. At $x=-28$ and $-18 \mathrm{~mm}$ (i.e., ahead of the slot location), downward shift of the location of $\tau_{\max }$ is consistent wit the increased mean velocities and velocity gradient nea the wall due to blowing. At $x=25$ and $39 \mathrm{~mm}$, the she stress profiles exhibit two peaks corresponding to the positive and negative maximum (normal) velocity grad ents which can be observed in the mean velocity profil (Fig. 5); these profiles resemble $2 \mathrm{D}$ wake flow with a vit locity minimum. A third peak in the shear stress which. to be expected close to the wall in the (jet) wall bound layer has not been captured in the measurements sinct occurs very lose to the wall $(y<0.5 \mathrm{~mm})$; in fact, at $x=39 \mathrm{~mm}$, measurement at the first $y$ location from $t$ wall reveals positive shear stress as expected.

The turbulent kinetic energy profiles at $x=-28$ and $-18 \mathrm{~mm}$ show features qualitatively similar to the shea stress profiles (Fig. 7); in particular, the downward shif the $y$ location corresponding to $k_{\max }$ may be seen. The increased $k$ levels near the wall at $x=25$ and $39 \mathrm{~mm}$ o viously arise form the increased (normal) velocity grad ents and $\tau$ levels in the attached boundary layer with blowing. Detailed analysis of the mean flow and turbult quantities with blowing from the point of view of turb lence modelling is currently in progress.

\subsection{5}

\section{Blowing requirements}

In the context of separation control, suppression of wa flow reversal may be adequate in certain applications ( relief in surface heat transfer at high speeds), while su pression of both wall and wake flow reversal may be portant in other cases (e.g. aircraft intakes). Estimates blowing mass flow and momentum coefficient for the 


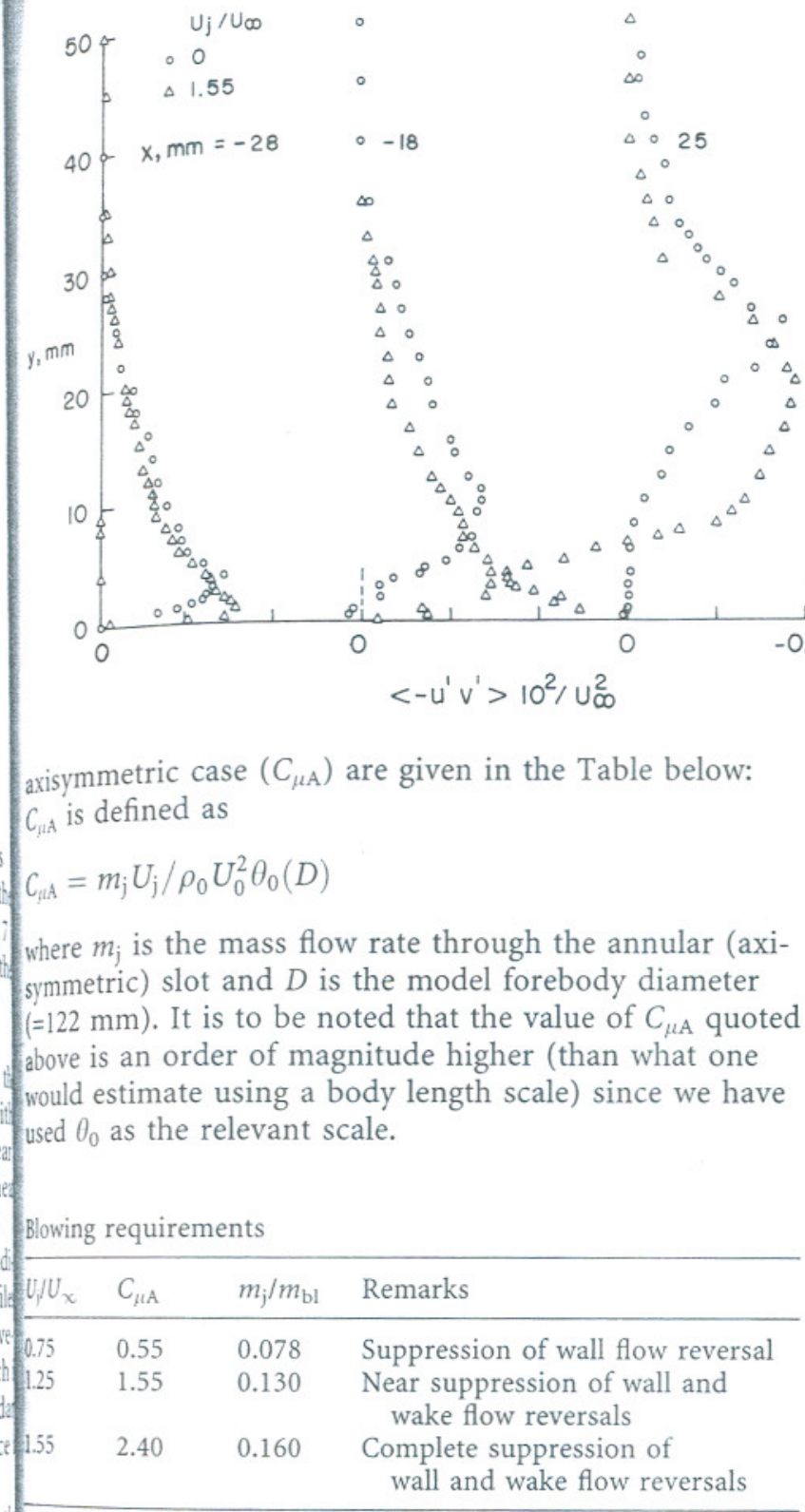

$\Delta \circ$

so 39

$\Delta \circ$

$\Delta$

$\triangle \quad \circ$

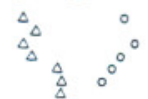

${ }_{\Delta}{ }_{\Delta}^{\circ}$

$\circ \Delta$

$\Delta \Delta$

Fig. 7. Effect of blowing on Reynolds shear stress profiles

\subsection{6}

Possible flow mechanisms associated with D-type blowing The effectiveness of $\mathrm{D}$-type blowing has been demonstrated and it is informative to speculate on possible flow mechanisms associated with it. In this technique, it is the separated bubble which is energized by the tangential jet. The wall flow reversal is first suppressed by the interaction of the jet (having higher total pressure or longitudinal momentum) with the (otherwise) reversed-flow boundary layer. Second, the fluid injection causes a strong mass imbalance in the bubble and altered shear layer entrainment characteristics. Finally, the jet entrainment of the reversed flow in the bubble is possibly a strong factor promoting increased mixing leading to higher (mean) kinetic energy levels near the wall. These features result in the removal or elimination of the shear layer reattachment.

4

\section{Conclusions}

It has been demonstrated through detailed flow measurements for the first time that tangential blowing through a

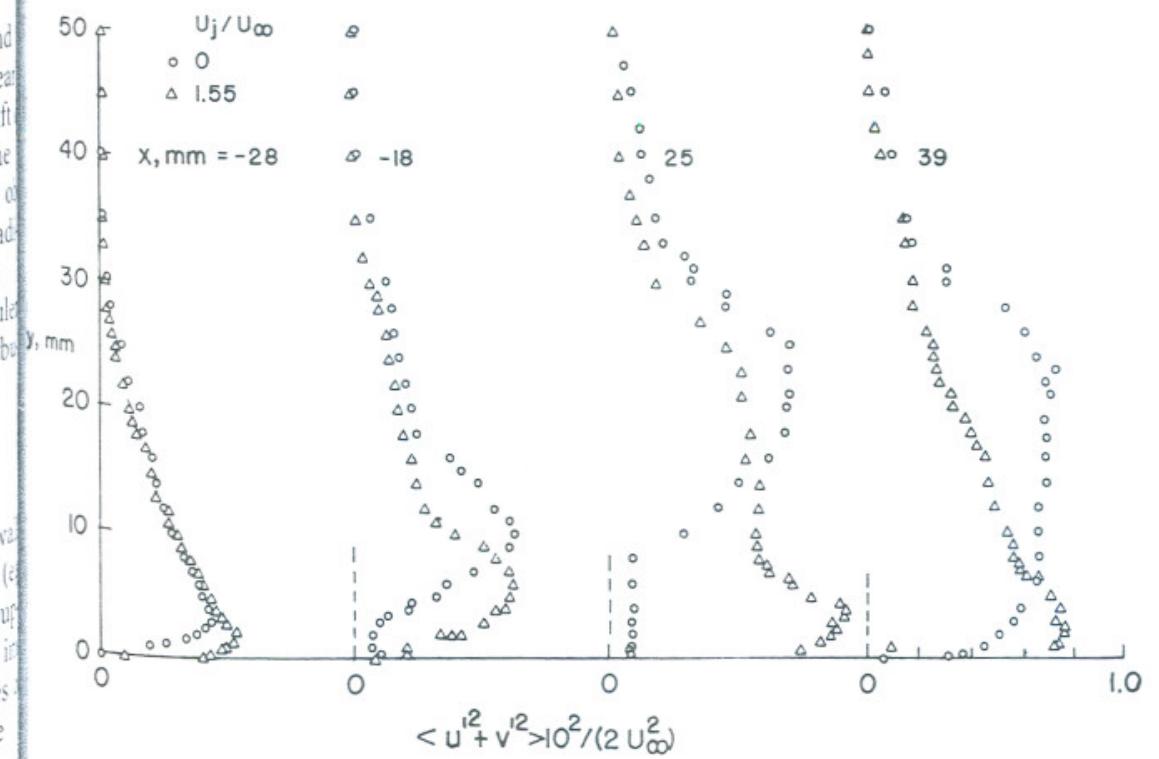

Fig. 8. Effect of blowing on turbulent kinetic energy profiles 
narrow slot downstream of separation, but within the bubble, can be an effective means of separation control. Dtype injection or blowing involves energizing the bubble flow leading to the elimination of shear layer reattachment, as opposed to energization of the boundary layer upstream of the separation point, which is the conventional technique adopted for boundary layer control. The mechanics of D-type blowing is therefore associated with manipulation of shear layer reattachment process which is a key element in the dynamics of separated flows as emphasized by Roshko $(1966,1967)$. Taken together with the success of D-type injection observed in a supersonic ramp flow (Viswanath et al., 1983), it may be concluded that D-type tangential blowing concept, although unconventional, may have significant potential for controlling separated flows in other situations (e.g. high-lift flows, shock-boundary layer interaction on supercritical airfoils). There is scope for optimizing the injection parameters for improved performance.

The experimental results presented in this paper also provide on excellent data base on an axisymmetric separated flow useful for improving turbulence modelling as well as CFD code validation.

\section{References}

Adams EW; Eaton JK (1988) An LDV study of the backward facing step flow, including the effects of velocity bias. J Fluids Eng 110: 275-282

Chang PK (1970) Separation of Flow, Pergamon Press

Chang PK (1976) Control of Flow Separation, Hemisphere Publishing Corporation

Gad-El-Hak; Bushnell DM (1991) Separation control: Review J Fluids Eng 113: 5-30

Lachmann GV Ed (1961) Boundary Layer and Flow Control, Vol. I, Pergamon Press

Marvin JG (1982) Turbulence modelling for computational aerodynamics, AIAA Paper 82-0164

Marvin JG (1983) Turbulent modelling of turbulent separated flows for aerodynamic applications, NASA TM 84392

Marvin JG (1995) Perspective on CFD validation, AIAA J 33: $1778-1787$

Peake DJ (1966) The use of air injection of prevent separation of the turbulent boundary layer in supersonic flow. Aeronautical Research Council (UK) report CP 890

Ramesh G; Madhavan KT; Viswanath PR (1995) LDV measurements in an axisymmetric separated flow, Part I: Without flow control, National Aerospace Laboratories Report PD EA 9505
Ramesh G; Madhavan KT; Geetha E; Kusuma Suresh (1994) based data acquisition and processing software for two pa $_{0}$ ponent Doppler Velocimetry. Paper presented at the 19 th National Symposium on Instrumentation, Tirupathi, Indit 23-24

Roshko A (1966) A look at our present understanding of rated flows. Paper presented at the AGARD Symposium Separated Flows, May 10-13, Brussels

Roshko A (1967) A review of concepts in separated flows. Pa presented at the Canadian Congress of Applied Mechanics, 22-26, Quebec, Canada

Sandborn VA; Liu CY (1968) On turbulent boundary layer aration, J Fluid Mech 32: 293-304

Simpson RL; Chew YT; Shivprasad BG (1981) The structure separating turbulent boundary layer, Part I: Mean flow Rey nolds stresses. J Fluid Mech 113: 23-51

Simpson RL (1985) Two-dimensional turbulent separated flow Agardograph 287

Simpson RL (1987) A review of two-dimensional separated fol calculation methods, In: Smith FT; Brown SN (eds). Proc TAM Symposium on Boundary Layer Separation 179-196 Simpson RL (1989) Turbulent boundary layer separation In: Lumley JL; Van Dyke M; Reed HL (eds) Ann Rev Fluid Mer Published by Annual Reviews Inc. USA, vol 21: 205-234

Stratford BS (1959) An experimental flow with zero skin fricti throughout its region of pressure rise. J Fluid Mech 5: 17-

Thompson BE; Whitelaw JH (1985) Characteristics of a trailit edge flow with turbulent boundary layer separation. J Fluid Mech 157: 305-326

Viswanath PR (1988) Shock wave-turbulent boundary layer it teraction and its control: A survey of recent developments Sadhana, Indian Academy Journal in Engineering Sciences 12: $45-104$

Viswanath PR; Brown JL (1983) Separated trailing-edge flow. AIAA J 21: 801-809

Viswanath PR; Sankaran L; Sagdeo PM; Narasimha R; Prabh (1983) Injection slot location for boundary-layer control in shock-induced separation. J Aircraft 20: 726-732

Weidemann J (1994) Laer Doppler Anemometrie, Springer Vet Berlin

Williams BR (1989) Viscous-inviscid interaction schemes for separated flow, In: Proc. of Symposium on the Prediction Exploitation of Separated Flow, Published by Royal Aeronautical Society, 5.1-5.16

Wong WF (1977) Application of boundary layer blowing to suppress strong shock-induced separation in supersonic in AIAA Paper $77-147$

Wygnanski I (1997) Boundary layer and flow control by peric addition of momentum. AIAA Paper 97-2117 